\title{
Risk Estimate
}

National Cancer Institute

\section{Source}

National Cancer Institute. Risk Estimate. NCI Thesaurus. Code C19015.

Methods to predict the degree of health risk arising from a specific health hazard. 